Schmerz 2010 $24: 207-208$

DOI 10.1007/s00482-010-0907-1

Online publiziert: 30 . April 2010

(c) Deutsche Gesellschaft zum Studium des Schmerzes. Published by Springer

Medizin Verlag - all rights reserved 2010

R.-D. Treede ${ }^{1,2} \cdot$ G. Müller-Schwefe ${ }^{3} \cdot$ R. Thoma ${ }^{4}$

${ }^{1}$ Neurophysiologie, Medizinische Fakultät Mannheim, Universität Heidelberg, Mannheim

${ }^{2}$ Deutsche Gesellschaft zum Studium des Schmerzes e.V. (DGSS), Boppard

${ }^{3}$ Deutsche Gesellschaft für Schmerztherapie e.V. (DGS), Oberursel

${ }^{4}$ Berufsverband der Schmerztherapeuten in Deutschland e.V. (BVSD), Berlin

\title{
Kodierung chronischer Schmerzen im ICD-10
}

Schmerzen. Umgekehrt verlangt der Kode für "somatoforme Schmerzstörung“ (F45.4), dass emotionale Konflikte oder psychosoziale Belastungen die Hauptrolle in der Genese des Schmerzes spielen müssen. Für die Mehrzahl der Patienten ist eine solche Dichotomisierung in primär psychisch vs. primär organisch bedingte Schmerzen nicht möglich. Dass für diese Patienten kein passender ICD-Code vorliegt, hat weitreichende Folgen:

Was nicht kodiert werden kann, - daran kann ein Patient nicht leiden.

Patienten, die keiner exakten Diagnose zugeordnet werden können, fühlen sich verlassen und hilflos, sind Selbstmordgedanken und Sterbehilfewünschen ausgesetzt. Sie dürfen nicht $\mathrm{Op}$ fer einer Untätigkeit und von berufspolitischen Rangeleien werden.

- das braucht nicht behandelt zu werden. Es herrscht ein Mangel an adäquat weitergebildeten Schmerztherapeuten. Eine schmerztherapeutische Versorgung in der Breite wird auf Dauer nur dann stattfinden, wenn die aufwendige Behandlung von Schmerzpatienten entsprechend dokumentiert und je nach Ausgestaltung eines neuen Vergütungssystems auch honoriert wird. Voraussetzung hierfür ist die Möglichkeit einer nach Schweregrad differenzierten Kodierung von Schmerz- und Palliativpatienten. Dies gilt sowohl für die hausärztliche als auch für die spezialisierte Versorgung. - dessen Behandlung braucht nicht gelehrt zu werden. In der Approbationsordnung von 2002 kommen
Schmerztherapie und Palliativmedizin nur im Anhang vor. Die Präambel der Weiterbildungsordnung geht davon aus, dass die allgemeine Schmerztherapie

von jedem Arzt beherrscht wird. In den einzelnen Fächern ist die allgemeine Schmerztherapie aber nicht verankert.

- dessen Kosten für das Gesundheitssystem werden nicht ermittelt. Für den morbiditätsbezogenen Risikostrukturausgleich können mit den bisherigen Kodes die Patienten mit chronifiziertem Schmerz nicht abgebildet werden, obwohl ihre Behandlung hohe Kosten verursacht. Wenn überhaupt, fallen sie mit dem Code F62.80 in die Kategorie „Schizophrenie“.

Die DGSS hat daher zusammen mit psychologischen, psychiatrischen und psychosomatischen Fachgesellschaften eine neue Diagnose Chronische Schmerzstörung mit Somatischen und Psychischen Faktoren formuliert [3], die zum Ausdruck bringt, dass psychische Faktoren oftmals eine wichtige Bedeutung im Chronifizierungsprozess haben und einen spezifischen Behandlungsbedarf auslösen, ohne jedoch eine monokausale Erklärung zu liefern.

$\mathrm{Zu}$ Beginn des Jahres 2008 wurden die DGSS, die DGS und der BVSD gemeinsam im Bundesgesundheitsministerium vorstellig, um noch einmal auf die eminente Wichtigkeit der korrekten Kodierung chronischer Schmerzen hinzuweisen. he von Kodes für rein. Es gibt eine Rei- 
Dank dieser konzertierten Aktion konnte im Laufe des Jahres 2008 die vorgeschlagene neue Diagnose mit dem DIMDI und den zuständigen Gremien so weit präzisiert werden, dass sie zum 01.01.2009 als F45.41 in die Deutsche Version des ICD10 aufgenommen wurde [2].

Mit der F45.41 ist erstmals eine angemessene Diagnose von Schmerzen mit gleichzeitig sensorischen, kognitiv-emotionalen und Verhaltensaspekten und damit von chronischen Schmerzen als komplexer biopsychosozialer Störung möglich. In engem Dialog mit der American Psychiatric Association soll diese Diagnose in Zukunft auch in die weltweit gültige ICD-11 und das DSM-V integriert werden.

In dieser Ausgabe der Zeitschrift „Der Schmerz" finden Sie jetzt eine Kodierhilfe zur Anwendung der neuen Diagnose F45.41 als Haupt- oder Nebendiagnose und zu deren Abgrenzung von anderen Schmerzdiagnosen [1]. Die Autoren gehören zu den Pionieren bei der Einführung der F45.41 und können daher bereits über erste praktische Erfahrungen berichten. Die Präsidenten von DGSS, DGS und BVSD sind davon überzeugt, dass durch den sachgerechten Einsatz dieser neuen Diagnose der Behandlungsbedarf für chronisch Schmerzkranke dokumentierbar ist (• Tab. 1). Mittelfristig wird dies neben einem genaueren Überblick über epidemiologische Daten auch der Qualität der Patientenversorgung zugute kommen.

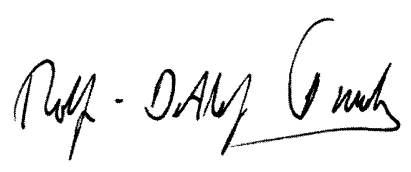

R.-D. Treede

\section{Korrespondenzadresse \\ Prof. Dr. R.-D. Treede}

Neurophysiologie, Medizinische Fakultät

Mannheim, Universität Heidelberg

Ludolf-Krehl-Str.13-17, 68167 Mannheim

rolf-detlef.treede@medma.uni-heidelberg.de

Danksagung. Die Autoren danken Herrn Dr. Michael Küster für die Argumentationshilfe gegenüber dem Bundesministerium für Gesundheit.
Tab. 1 Chronische Schmerzstörung mit somatischen und psychischen Faktoren (ICD-10 GM Version 2009: F45.41)

Monokausale somatische Selten möglich Attribution:

Monokausal psycholo- Selten möglich gische Attribution:

Chronischer Schmerz als Krankheit:

- Verlust der Warnfunktion (nicht operationalisiert)

- Psychologische Faktoren sind relevant (und benötigen Therapie)

Relevanz des ICD-Kodes:

- Zusätzlicher Behandlungsbedarf für chronische Schmerzen kodierbar

- Epidemiologie

- Vergütung im stationären Sektor (DRG)

- Morbiditätsbezogener Risikostrukturausgleich zwischen den Kassen

\section{Literatur}

1. Nilges P, Rief W (2010) F45.41 Chronische Schmerzstörung mit somatischen und psychischen Faktoren - eine Kodierhilfe. Schmerz 24, DOI 10.1007/ s00482-010-0908-0

2. Rief $W$, Treede RD, Schweiger U, Henningsen $P$, Rüddel H, Nilges P (2009) Neue Schmerzdiagnose in der deutschen ICD-10-Version. Nervenarzt 80:340-342

3. Rief W, Zenz M, Schweiger U et al (2008) Redefining (somatoform) pain disorder in ICD-10: a compromise of different interest groups in Germany. Current Opinion Psychiatry 21:178-181

4. Treede RD (2007). Entstehung der Schmerzchronifizierung. In: Baron R, Strumpf M (Hrsg) Praktische Schmerztherapie. Springer, Berlin Heidelberg New York Toyko, S 3-12

\section{Wolffram-Preis für Forschungsarbeiten zum Thema Kopfschmerz}

Ausschreibung der Deutschen Migräne- und Kopfschmerzgesellschaft (DMKG)

Eingereicht werden können Diplomarbeiten, Doktorarbeiten und Publikationen (Publikationen sollten zumindest ,im Druck" sein bzw. nicht älter als 12 Monate). Für den Preis können sich wissenschaftliche Mitarbeiterinnen und Mitarbeiter aller Fachrichtungen bewerben, die an einer akademischen Einrichtung in Deutschland tätig sind. Es besteht keine Altersbegrenzung.

Der erste Preis wird mit $5.000 €$, der zweite mit $2.500 €$ und der dritte mit $1.000 €$ gewürdigt.

Die Bewertung nimmt das DMKG-Präsidium vor. Dieses behält sich vor, externe Gutachter hinzuziehen.

Der Wolffram-Preis wird anlässlich des Deutschen Schmerzkongresses vom 6. - 9.10. 2010 in Mannheim verliehen. Bewerbungen sind in vierfacher Ausfertigung bis zum 15. August 2010 Poststempel) zu richten an:

PD Dr. Stefanie Förderreuther Generalsekretärin der DMKG Neurologische Klinik der Universität München Konsiliardienst am Standort Innenstadt Ziemssenstrasse 1 80336 München 